\title{
27-Meter-Long Ultra-Low-Loss Optical Delay Line on a Silicon Chip
}

\author{
Tong Chen, Hansuek Lee, Jiang Li, Oskar Painter, and Kerry Vahala \\ Thomas J. Watson, Sr., Laboratory of Applied Physics, California Institute of Technology, \\ 1200 E. California Boulevard,Pasadena, CA 91125, USA \\ vahala@caltech.edu
}

\begin{abstract}
Using a wet etch process, 27 meter long waveguides having optical loss of less than $0.1 \mathrm{~dB} / \mathrm{m}$ are demonstrated. Resonator measurements show that this loss value can be reduced to $0.037 \mathrm{~dB} / \mathrm{m}$

(C) 2011 Optical Society of America

OCIS codes: (230.7370) Waveguide; (060.2370) Fiber optics sensors; (130.2755) Glass waveguides.
\end{abstract}

A multi order-of-magnitude gap exists between the loss of optical fiber and chip-based photonic waveguides. Most of the loss is not fundamental and is instead caused by process-induced surface roughness. Even partially closing this gap could benefit applications in sensing, radio-frequency photonics and signal processing. The question of reducing optical loss in photonic circuits has been considered for some time [1] and there has been considerable progress [2, 3]. In this work, a process previously used to fabricate high-Q resonators in silica [4], has been modified to create 27 meter long delay lines with a loss of $0.1 \mathrm{~dB} / \mathrm{m}$ as measured by optical backscatter reflectometry. These are the longest and lowest loss waveguides ever fabricated. Additionally, losses as low as $0.037 \mathrm{~dB} / \mathrm{m}$ are inferred from resonator optical Q measurements.
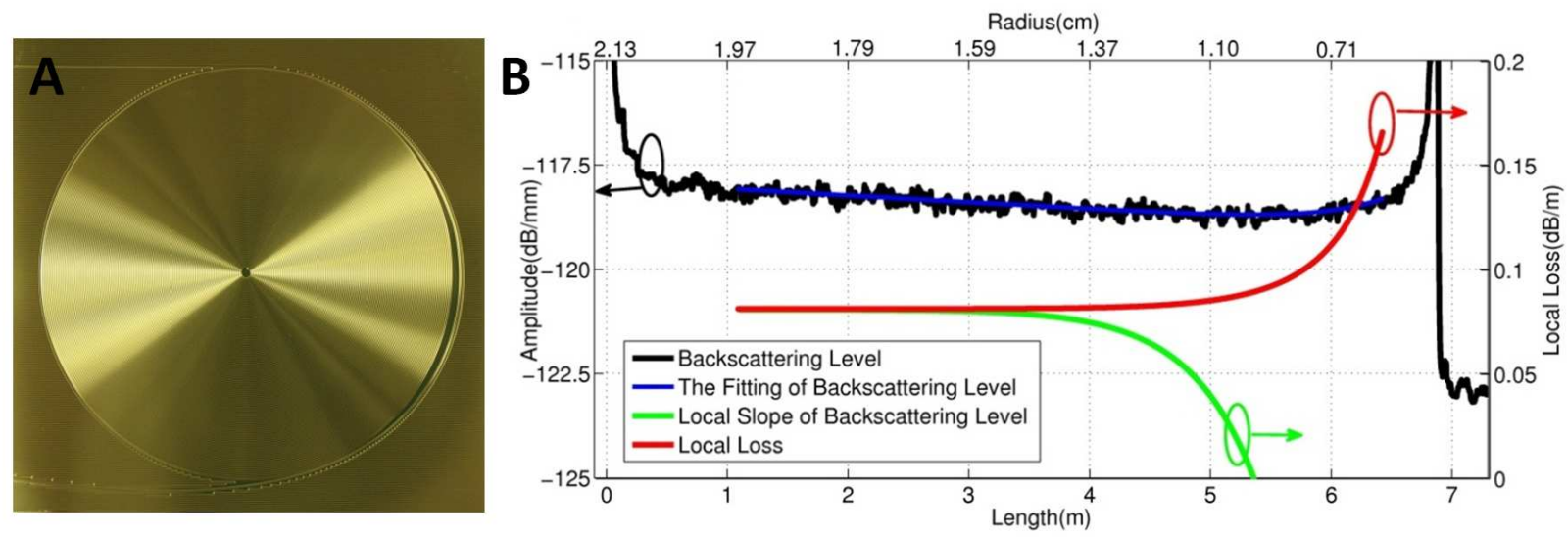

Fig. 1. (A) An optical micrograph of a 7 meter physical path length, one-way spiral with a diameter of $4.3 \mathrm{~cm}$. (B) Optical backscatter reflectometer measurement of the spiral waveguide shown in panel A. On account of the return path, the backscatter decay rate is increased by $2 \times$. The blue curve is a fit to the backscatter level, while the green curve gives a fit to the local backscatter decay rate and the red curve uses a model to infer the actual local waveguide attenuation, which is approximately $0.08 \mathrm{~dB} / \mathrm{m}$ over most of the spiral. The upper axis gives the radius from center while the lower axis gives the physical path length.

In cross section the silica waveguides have a wedge profile that is air-clad with the mode guided in the wedge region and mechanical support provided by a silicon pillar at the interior [4]. Figure 1A shows a single spiral with a path length of approximately 7 meters. Backscatter measurements using a Luna OBR 4400 are presented in figure 2B and a linear decrease is observed (on the log scale) over nearly the full 7 meters of the spiral path. The inferred loss rate from this data is $0.05 \mathrm{~dB} / \mathrm{m}$ and provides a lower bound on the waveguide attenuation rate. To refine the estimate, the impact of the varying backscatter signal with variation of the spiral radius can be calibrated using a model. A corrected value of $0.08 \mathrm{~dB} / \mathrm{m}$ for the loss of the device in figure $1 \mathrm{~A}$ is then obtained.

Figure 2A shows four spirals cascaded on a single wafer to create a $27 \mathrm{~m}$ (physical length) waveguide. Each spiral is approximately $4 \mathrm{~cm}$ in diameter and features inward and outward, interlaced Archimedean-shaped waveguides that are connected at the spiral center by an adiabatic coupler. The adiabatic coupler features a completely undercut silica guide that is designed so as to maintain propagation in the fundamental transverse mode through the clockwise to 

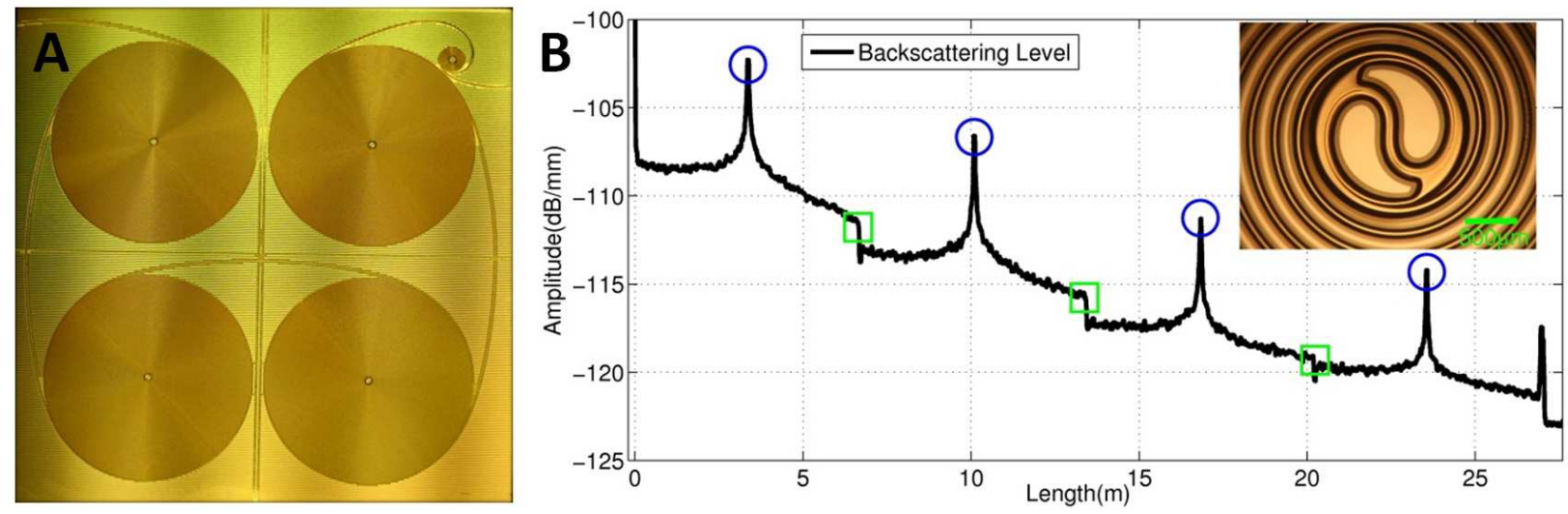

Fig. 2. (A) Optical micrograph of a cascaded, four-spiral waveguide having a physical path length of 27 meters. The input port is in the upper left of the image, and the waveguides connecting neighboring spiral delays are visible. The entire chip is $9.5 \mathrm{~cm} \times 9.5 \mathrm{~cm}$. (B) Optical backscatter reflectometer measurement of the spiral waveguide shown in panel $\mathbf{A}$. The periodic singularities (blue circles) in the backscatter signal correspond to the optical wave transiting the inner adiabatic coupling region of each spiral. Other discontinuities (green squares) in back scatter occur when the optical wave transfers between the spirals and result from higher order transverse modes being mode filtered. The inset is a magnified view of the adiabatic coupling section, which is approximately $1 \mathrm{~mm}$ in diameter.

counter-clockwise turn. Analysis of waveguide attenuation in spirals 3 and 4 gives approximately $0.1 \mathrm{~dB} / \mathrm{m}$ for the waveguide loss and insertion loss of less then $0.1 \mathrm{~dB} / \mathrm{m}$ for the adiabatic coupler. Spirals 1 and 2 show an apparent waveguide loss of $0.2 \mathrm{~dB} / \mathrm{m}$, that is attributed to the presence of higher order radial modes. These modes mask the fundamental mode in the observed backscatter signal until they are filtered through their higher level of attenuation. The slight difference in optical loss $(0.08 \mathrm{~dB} / \mathrm{m}$ vs. $0.1 \mathrm{~dB} / \mathrm{m})$ for the spirals of figure $1 \mathrm{~A}$ and $2 \mathrm{~A}$ might reflect some slight process variation in the fabrication of these devices.

To obtain the dependence of attenuation on waveguide bending, the $\mathrm{Q}$ factor of disk resonators of varying diameters is measured. Data in figure 3 show that optical attenuation as low is $0.037 \mathrm{~dB} / \mathrm{m}$ can be obtained in these structures, corresponding to an optical Q factor of 670 million. AFM measurements of the upper, wedge and lower surfaces have also been performed on these resonators giving corresponding rms roughness variances of $0.15 \mathrm{~nm}$ and $0.48 \mathrm{~nm}$ with correlation length of approximately $200 \mathrm{~nm}$ for the upper and wedge surfaces, respectively. The roughness of the lower surface is as large as $1-2 \mathrm{~nm}$ in amplitude and has been used as a fitting parameter to model the loss versus diameter in figure 3. Also, a projection of the predicted loss is given assuming that the lower surface roughness could be reduced to that of the upper oxide surface. In the current design, loss levels lower than $0.01 \mathrm{~dB} / \mathrm{m}$ could be realized.

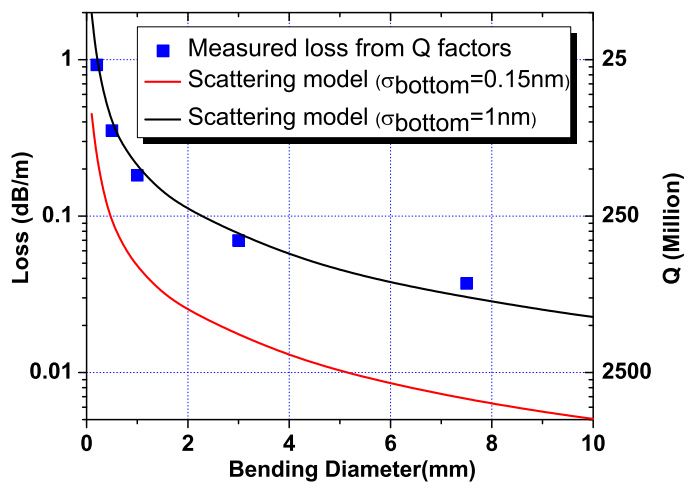

Fig. 3. Bending loss data obtained from resonator Q measurements plotted versus the bending diameter. The black curve is a fit to the data using roughness data provided in the text and obtained using an AFM. The principle source of roughness is the lower dielectric interface and an estimate of the bending loss in case this surface can be smoothed to the level of the upper surface is provided as the red curve.

\section{References}

1. R. Adar, M. Serbin, and V. Mizrahi, "Less than $1 \mathrm{~dB}$ per meter propagation loss of silica waveguides measured using a ring resonator," J. of Light. Tech. 12, 1369-1372 (1994).

2. T. Kominato, Y. Hida, M. Itoh, H. Takahashi, S. Sohma, T. Kitoh, and Y. Hibino, "Extremely low-loss (0.3 dB/m) and long silica-based waveguides with large width and clothoid curve connection," in Proceedings of ECOC (Stockholm, Sweden, 2004).

3. J. F. Bauters, M. J. R. Heck, D. John, D. Dai, J. S. Barton, C. M. Bruinink, A. Leinse, R. G. Heideman, D. J. Blumenthal, and J. E. Bowers, "Planar waveguides with less than $0.1 \mathrm{~dB} / \mathrm{m}$ propagation loss fabricated with wafer bonding," Optics Express, 19, 24090-24101 (2011).

4. T. J. Kippenberg, J. Kalkman, A. Polman, and K. J. Vahala, "Demonstration of an erbium-doped microdisk laser on a silicon chip," Physical Review A, 74, 051802 (2006). 